\title{
Stable minimal surfaces in Riemannian warped products
}

\author{
Juan A. Aledo ${ }^{a}$ and Rafael M. Rubio ${ }^{b}$ \\ ${ }^{a}$ Departamento de Matemáticas, E.S.I. Informática, \\ Universidad de Castilla-La Mancha, 02071 Albacete, Spain, \\ E-mail: juanangel.aledo@uclm.es \\ ${ }^{b}$ Departamento de Matemáticas, Campus de Rabanales, \\ Universidad de Córdoba, 14071 Córdoba, Spain, \\ E-mail: rmrubio@uco.es
}

\begin{abstract}
We obtain several stability results for minimal two-sided surfaces immersed in a wide class of 3-dimensional Riemannian warped products, which includes the class of Riemannian products. As a consequence, some Bernstein-type results are provided. Moreover, a nonexistence theorem for a certain non-linear elliptic equation is obtained.
\end{abstract}

Mathematical Subject Classification: 53A10, 58J05.

Keywords: Minimal stable surface, Riemannian warped product, Bernstein problem.

\section{Introduction}

There is an important subclass of minimal surfaces that contains that of area-minimizing surfaces: the class of stable minimal surfaces. Basically, a stable surface is area-minimizing relative to nearby surfaces with the same boundary. In variational terms, minimal surfaces are critical points of the area functional for compactly supported normal variations. Then, a minimal surface is stable if the second derivative of the area functional is non-negative for such normal variations. However, if for some deformation the second variation is negative, then there are nearby surfaces of smaller area, and the surface is called unstable.

Stable surfaces have become a fashion research topic which has received many interesting contributions in the last decades. Thus, Barbosa and Do Carmo [2] proved that for a surface $\Sigma$ in $\mathbb{R}^{3}$, if the area of the spherical image $N(D) \subset \mathbb{S}^{2}$ of a domain $D \subset \Sigma$ is smaller than $2 \pi$, then $D$ is stable. The result above provides a sufficient condition for a surface to be stable, but also the assumption of stability has significant consequences. For instance, Schoen [13] obtained a bound for the Gaussian curvature of stable surfaces which allows to obtain important structural consequences 
for the surface. Thus, from the mentioned inequality it can be concluded that every complete stable minimal surface immersed a 3-dimensional Riemannian manifold with non-negative Ricci curvature is totally geodesic [13], and that the stable complete surfaces in $\mathbb{R}^{3}$ are planes [5]. On the other hand, Shoen and Yau used the existence of certain area-minimizing surfaces to obtain topological obstructions to the existence of metrics with positive scalar curvature [14], [15].

More recently, Meeks and Rosenberg have classified the properly embedded minimal surfaces in $M \times \mathbb{R}$, where $M$ is a compact Riemannian surface [10].

As well-known, in the classical paper [4] Bishop and O'Neill introduced the notion of warped product manifold, which widely generalizes the usual product of Riemannian manifolds. In this way, they obtained a large class of Riemannian manifolds with negative curvature (see also [12, Chapter 7]).

In this work we deal with the stability of minimal surfaces immersed in a 3-dimensional Riemannian warped product $I \times_{f} M$, where $\left(M, g_{M}\right)$ is a (connected) complete Riemannian surface, $I \subseteq \mathbb{R}$ an open interval in $\mathbb{R}$ endowed with the metric $d t^{2}$, and $f>0$ a smooth function on $I$. We will assume that the warping function $f$ is convex, i.e., with non negative second derivative (see Section 3.1 for the details). This is a natural assumption which was already considered by Bishop and O'Neill in their aforementioned primigenius definition. Of course, this setting widely generalizes the one of standard Riemannian products $(f=1)$.

The paper is organized as follows. In Section 2, we generalize a classical characterization due to Fischer-Colbrie and Schoen [7] for stable minimal surfaces immersed in a 3-dimensional Riemannian manifold (Theorem 1). In Section 3.1 we revise some basic facts for surfaces immersed in a 3dimensional Riemannian warped product, and give some technical results. We devote Section 3.2 to provide our main results. Thus, we first prove that every minimal two-sided surface with positive angle function $\nu$ (which is satisfied, in particular, when the surface is locally a graph) immersed in a 3-dimensional Riemannian warped product $I \times{ }_{f} M$ with $f^{\prime \prime} \geq 0$ is stable (Theorem 4 and Corollary 5 ). Letting $\nu$ be non negative instead of positive, we provide several conditions for a minimal twosided surface to be either stable or be contained in a minimal cylinder (Theorems 6 and 9). When the ambient space has non-negative Ricci curvature, we get some Bernstein-type results thanks to a well-known result due to Schoen [13], so giving sufficient conditions for a minimal two-sided surface to be totally geodesic (see Theorems 10 and 11). As a consequence of this study, we get a non-existence result for the nonlinear elliptic equation of minimal graphs in $I \times_{f} M$ when $f$ is linear (Theorem 13). Finally, we provide some specific results for the particular case when the fiber is compact (Section 3.3) and when the 3-dimensional Riemannian warped product is Einstein (Section 3.4).

\section{$2 \quad$ A first general result}

Let $\Sigma$ be a surface immersed in a 3-dimensional Riemannian manifold $(\bar{M}, \bar{g})$ and let us denote by $\langle$,$\rangle the induced metric on \Sigma$. Let us suppose that $\Sigma$ is two-sided, i.e., its normal bundle is trivial and so there is a unit normal vector field $N$ globally defined on $\Sigma$. Take $A$ the shape operator associated to $N$. 
Provided that $\Sigma$ is minimal, its stability is determined by means of the quadratic form

$$
Q(\eta, \eta)=-\int_{\Sigma}\left(\triangle \eta+|A|^{2} \eta+\overline{\operatorname{Ric}}(N, N) \eta\right) \eta, \quad \eta \in \mathcal{C}_{0}^{\infty}(\Sigma),
$$

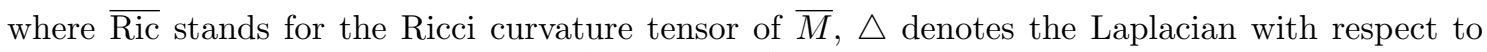
the induced metric $\langle$,$\rangle on \Sigma$ and $|A|^{2}=\operatorname{trace}\left(A^{2}\right)$ (see [6]). Observe that, from the divergence theorem,

$$
\int_{\Sigma} \eta \triangle \eta=-\int_{\Sigma}|\nabla \eta|^{2}, \quad \eta \in \mathcal{C}_{0}^{\infty}(\Sigma)
$$

where $\nabla$ denotes the gradient with respect to $\langle$,$\rangle , and so Q$ can be rewritten as

$$
Q(\eta, \eta)=\int_{\Sigma}\left(|\nabla \eta|^{2}-\left(|A|^{2}+\overline{\operatorname{Ric}}(N, N)\right) \eta^{2}\right), \quad \eta \in \mathcal{C}_{0}^{\infty}(\Sigma) .
$$

In fact, the minimal surface $\Sigma$ is stable if, and only if, $Q(\eta, \eta) \geq 0$ for all $\eta \in \mathcal{C}_{0}^{\infty}(\Sigma)$.

On the other hand, Fischer-Colbrie and Schoen proved in [7] that the condition above is equivalent to the existence of a positive function $u \in \mathcal{C}^{\infty}(\Sigma)$ such that $L u=0$ for the Jacobi operator

$$
L=\triangle+|A|^{2}+\overline{\operatorname{Ric}}(N, N) \text {. }
$$

Our aim in this section is to generalize this last result as follows:

Theorem 1 Let $\Sigma$ be a minimal surface immersed in a 3-dimensional Riemannian manifold $(\bar{M}, \bar{g})$. Then $\Sigma$ is stable if, and only if, there exists a positive function $u \in \mathcal{C}^{\infty}(\Sigma)$ such that $L u \leq 0$.

Proof: Bearing in mind the result by Fischer-Colbrie and Schoen, it is enough to prove that if there exists a positive function $u \in \mathcal{C}^{\infty}(\Sigma)$ satisfying that $L u \leq 0$, then $\Sigma$ is stable.

Thus, let us assume that there exists such a function $u$ and take $\eta \in \mathcal{C}_{0}^{\infty}(\Sigma)$. Observe that we can put $\eta=\varphi u$ for a certain $\varphi \in \mathcal{C}_{0}^{\infty}(\Sigma)$.

Using (2) we have that

$$
\int_{\Sigma}|\nabla \eta|^{2}=-\int_{\Sigma} \varphi u \triangle(\varphi u)=-\int_{\Sigma}\left(\varphi^{2} u \triangle u+\varphi u^{2} \triangle \varphi+2 \varphi u\langle\nabla \varphi, \nabla u\rangle\right) .
$$

Then, since $L u \leq 0$ we get from (3) that

$$
\begin{aligned}
Q(\eta, \eta) & =-\int_{\Sigma}\left[\varphi^{2} u\left(\triangle u+\left(|A|^{2}+\overline{\operatorname{Ric}}(N, N)\right) u\right)+\varphi u^{2} \triangle \varphi+2 \varphi u\langle\nabla \varphi, \nabla u\rangle\right] \\
& \geq-\int_{\Sigma}\left(\varphi u^{2} \triangle \varphi+2 \varphi u\langle\nabla \varphi, \nabla u\rangle\right)=-\int_{\Sigma}\left(\frac{1}{2}\left\langle\nabla \varphi^{2}, \nabla u^{2}\right\rangle+\varphi u^{2} \triangle \varphi\right) \\
& =\int_{\Sigma}|\nabla \varphi|^{2} u^{2} \geq 0,
\end{aligned}
$$

which finishes the proof. Above we have also used that

$$
\operatorname{div}\left(u^{2} \nabla \varphi^{2}\right)=\left\langle\nabla \varphi^{2}, \nabla u^{2}\right\rangle+u^{2} \triangle \varphi^{2}=\left\langle\nabla \varphi^{2}, \nabla u^{2}\right\rangle+u^{2}\left(2 \varphi \triangle \varphi+2|\nabla \varphi|^{2}\right)
$$

and the divergence theorem. 


\section{Surfaces in Warped Products}

\subsection{Set up}

Let $\left(M, g_{M}\right)$ be a (connected) complete Riemannian surface, $I \subseteq \mathbb{R}$ an open interval in $\mathbb{R}$ endowed with the metric $d t^{2}$, and $f>0$ a smooth function on $I$. We will denote by $\bar{M}=I \times{ }_{f} M$ the product $I \times M$ endowed with the Riemannian metric

$$
\bar{g}=-\pi_{I}^{*}\left(d t^{2}\right)+f\left(\pi_{I}\right)^{2} \pi_{M}^{*}\left(g_{M}\right)
$$

where $\pi_{I}$ and $\pi_{M}$ denote the projections onto $I$ and $M$ respectively. Then, $(\bar{M}, \bar{g})$ is a Riemannian warped product with fiber $\left(M, g_{M}\right)$, base $\left(I,-d t^{2}\right)$ and warping function $f$ (see [12, Chapter 7]).

We will denote by $T=\bar{\nabla} t$ the gradient of $t:=\pi_{I}$ for the metric $\bar{g}$. In $\bar{M}$ we will consider the closed conformal vector field $\xi:=f(t) T$. In fact, from the relationship between the Levi-Civita connections of $\bar{M}$ and those of $\left(M, g_{M}\right)$ and $\left(I, d t^{2}\right)$ [12, Cor. 7.35], it follows that

$$
\bar{\nabla}_{X} \xi=f^{\prime}(t) X
$$

for any $X \in \mathfrak{X}(\bar{M})$, where $\bar{\nabla}$ is the Levi-Civita connection of the metric (5).

Let $\psi: \Sigma \longrightarrow \bar{M}$ be an isometric immersion of a surface $\Sigma$ in $\bar{M}$ and let us denote by $\langle$,$\rangle the$ induced metric on $\Sigma$.

Throughout this paper we will assume that $\Sigma$ is two-sided, i.e., there exists a unit normal vector field $N$, the Gauss map of $\psi$, globally defined on $\Sigma$. Moreover, we will assume that the angle function $\nu=\bar{g}(T, N)$ does not change sign on $\Sigma$. Specifically, we will take $N$ such that $\nu \geq 0$ on $\Sigma$. In particular, note that if $\Sigma$ is a local graph, then the angle function does not change sign on $\Sigma$; actually, it is $\nu>0$.

The family of surfaces $\Sigma_{t}=\{t\} \times M, t \in I$, constitutes a foliation of $\bar{M}$ by complete totally umbilical leaves of constant mean curvature $(\log f)^{\prime}(t)=\frac{f^{\prime}(t)}{f(t)}$ that we will call slices. Note that the condition $(\log f)^{\prime \prime}(t) \geq 0$ means that the mean curvature of the slices is non-decreasing. Observe that the condition $f^{\prime \prime}(t) \geq 0$, that we will use later, is weaker than $(\log f)^{\prime \prime}(t) \geq 0$. As commented in the Introduction, the assumption $f^{\prime \prime} \geq 0$ was initially considered by Bishop and O'Neill when they introduce the notion of Riemannian warped product [4].

We will say that a surface $\psi: \Sigma \longrightarrow \bar{M}$ is contained in a slab if it is contained between two slices. In other words, the height function $\tau=t \circ \psi$ is such that $\tau(\Sigma) \subseteq\left[t_{1}, t_{2}\right]$ for $t_{1}, t_{2} \in I$, $t_{1}<t_{2}$. Observe also that a surface $\psi: S \longrightarrow \bar{M}$ is contained in a slice if, and only if, $\nu \equiv 1$ along $\psi$.

Next we compute the operator $L$ given in (4) on the distinguished function $\bar{g}(\xi, N)$ defined on $\Sigma$. First, from (6) the gradient of $\tau$ on $\Sigma$ can be calculated to obtain

$$
\nabla \tau=T^{\top}=T-\nu N
$$

where by ()$^{\top}$ we mean taking the tangential component of a vector field along $\psi$, and so

$$
\nabla \bar{g}(\xi, N)=-f(\tau) A\left(T^{\top}\right)
$$


where $A$ is the shape operator associated to $N$. Then the Laplacians of $\tau$ and $\bar{g}(\xi, N)$ on $\Sigma$ become, respectively,

$$
\triangle \tau=\frac{f^{\prime}(\tau)}{f(\tau)}\left(1+\nu^{2}\right)+2 H \nu
$$

and

$$
\begin{aligned}
\triangle \bar{g}(\xi, N)= & -2 f(\tau) \bar{g}(\nabla H, \nabla \tau)-\bar{g}(\xi, N)\left(1-\nu^{2}\right)\left(\frac{K^{M} \circ \pi_{M}}{f(\tau)^{2}}+(\log f)^{\prime \prime}(\tau)\right) \\
& -2 f^{\prime}(\tau) H-\bar{g}(\xi, N)|A|^{2},
\end{aligned}
$$

where $H$ is the mean curvature of $\Sigma, K^{M}$ the Gaussian curvature of $M$ and $|A|^{2}=\operatorname{trace}\left(A^{2}\right)$.

We will also use that, for a minimal surface $(H \equiv 0)$, we have

$$
|\nabla \bar{g}(\xi, N)|^{2}=\frac{1}{2}|A|^{2}\left(f(\tau)^{2}-\bar{g}(\xi, N)^{2}\right) .
$$

In fact, let us take a local orthonormal frame $\left\{E_{1}, E_{2}\right\}$ on $\Sigma$ with $A E_{1}=\mu E_{1}$ and $A\left(E_{2}\right)=-\mu E_{2}$ for a certain function $\mu$. If we put $T^{\top}=k_{1} E_{1}+k_{2} E_{2}$ for certain functions $k_{1}$ and $k_{2}$, then

$$
\bar{g}\left(A T^{\top}, A T^{\top}\right)=\mu^{2}\left(k_{1}^{2}+k_{2}^{2}\right)=\frac{1}{2}|A|^{2}\left|T^{\top}\right|^{2}=\frac{1}{2}|A|^{2}\left(1-\nu^{2}\right),
$$

which jointly with (7) allow to obtain (9).

Observe that the Ricci curvature $\overline{\text { Ric }}$ of $\bar{M}$ can be expressed in terms of the Ricci curvature $\mathrm{Ric}_{\mathrm{M}}$ of $M$ (see [12, Cor 7.43]) as

$$
\overline{\operatorname{Ric}}(V, W)=\operatorname{Ric}_{\mathrm{M}}\left(V^{M}, W^{M}\right)-\left(2 \frac{f^{\prime}(t)^{2}}{f(t)^{2}}+(\log f)^{\prime \prime}(t)\right) \bar{g}(V, W)-(\log f)^{\prime \prime}(t) \bar{g}(V, T) \bar{g}(W, T),
$$

where $V, W \in \mathfrak{X}(\bar{M})$ and $V^{M}=V-\bar{g}(V, T) T, W^{M}=W-\bar{g}(W, T) T$ are their projections on $M$.

In particular, we have that

$$
\overline{\operatorname{Ric}}(N, N)=\left(1-\nu^{2}\right)\left(\frac{K^{M} \circ \pi_{M}}{f(\tau)^{2}}+(\log f)^{\prime \prime}(\tau)\right)-2 \frac{f^{\prime \prime}(\tau)}{f(\tau)} .
$$

With all of this, we get

Lemma 2 Let $\Sigma$ be a minimal two-sided surface immersed in a 3-dimensional Riemannian warped product $\bar{M}=I \times_{f} M$. Then

$$
L \bar{g}(\xi, N)=-2 \frac{f^{\prime \prime}(\tau)}{f(\tau)} \bar{g}(\xi, N)
$$

Next, we compute the Gaussian curvature $K$ of $\Sigma$. If we denote by $\overline{\mathcal{S}}$ the scalar curvature of $\bar{M}$, it is a straightforward computation (see [1, Section 2]) to check that

$$
K=\frac{1}{2} \overline{\mathcal{S}}-\overline{\operatorname{Ric}}(N, N)+2 H^{2}-\frac{1}{2}|A|^{2} .
$$


Furthermore, we have that (see [12, Ex. 7.13])

$$
\overline{\mathcal{S}}=\frac{K^{M}}{f(t)^{2}}-4 \frac{f^{\prime \prime}(t)}{f(t)}-2 \frac{f^{\prime}(t)^{2}}{f(t)^{2}} .
$$

Finally, from (12), (13), (11) and using also the Gauss and Codazzi equations of the isometric immersion $\psi: \Sigma \longrightarrow \bar{M}$, we get

$$
K=-\frac{f^{\prime}(\tau)^{2}}{f(\tau)^{2}}-\left(1-\nu^{2}\right)\left(\frac{K^{M} \circ \pi_{M}}{f(\tau)^{2}}+(\log f)^{\prime \prime}(\tau)\right)+\frac{K^{M} \circ \pi_{M}}{f(\tau)^{2}}+2 H^{2}-|A|^{2} .
$$

To end this section, we will describe a special family of minimal surfaces which will play an important role later

Example 3 Given $J \subseteq I$ and $\gamma: \widetilde{J} \longrightarrow M$ a smooth curve defined in a certain real interval $\widetilde{J}$, consider the cylinder

$$
C(J, \gamma)=\{(t, \gamma(s): t \in J, s \in \widetilde{J})\} \subseteq I \times_{f} M .
$$

Observe that $C(J, \gamma)$ is minimal if, and only if, $\gamma$ is a totally geodesic curve in $M$.

On the other hand, given an immersion $\psi: \Sigma \longrightarrow \bar{M}$ with Gauss map $N$, it is easy to check that the angle $\nu$ vanishes identically on $\Sigma$ (or equivalently $\bar{g}(\xi, N) \equiv 0$ on $\Sigma$ ) if, and only if, $\psi(\Sigma)$ is contained in a cylinder $C(J, \gamma)$ for a certain $\gamma: \widetilde{J} \longrightarrow M$.

\subsection{Main Results}

As a direct consequence of Theorem 1 and Lemma 2, we have the following two results for surfaces with positive angle $\nu>0$ (and so, in particular, for local graphs).

Theorem 4 Let $\Sigma$ be a minimal two-sided surface immersed in a 3-dimensional Riemannian warped product $\bar{M}=I \times_{f} M$. If $\nu>0$ and $f^{\prime \prime}(\tau) \geq 0$ on $\Sigma$, then $\Sigma$ is stable.

In particular we have

Corollary 5 Let $\bar{M}=I \times_{f} M$ be a 3-dimensional Riemannian warped product whose warping function satisfies that $f^{\prime \prime} \geq 0$. Then every minimal two-sided surface with $\nu>0$ is stable.

The hypothesis on the angle function can be weaken, letting $\nu$ be non negative, to obtain the following result

Theorem 6 Let $\Sigma$ be a minimal two-sided surface immersed in a 3-dimensional Riemannian warped product $\bar{M}=I \times_{f} M$, with $\nu \geq 0$. If $f^{\prime \prime}(\tau)=0$ on $\Sigma$, then either $\nu>0$ (and so $\Sigma$ is stable) or $\nu$ vanishes identically on $\Sigma$ and so $\psi(\Sigma)$ is contained in a minimal cylinder $C(J, \gamma)$.

Proof: Under the assumptions of the theorem, we know that the Jacobi operator $L$ satisfies that $L u \equiv 0$ for the non negative function $u=\bar{g}(\xi, N)$. Let us assume that there exists a point $p_{0} \in \Sigma$ where $u\left(p_{0}\right)=0$ and take $\mathcal{V}\left(p_{0}\right)$ a relatively compact open neighborhood of $p_{0}$ in $\Sigma$. 
Let us take

$$
m=\min \left\{0, \inf _{\mathcal{V}\left(p_{0}\right)}\left(|A|^{2}+\overline{\operatorname{Ric}}(N, N)\right)\right\} .
$$

Then, for the elliptic operator $L^{\prime}=\triangle+m$ we have that $L^{\prime}(-u) \geq 0$ on $\mathcal{V}\left(p_{0}\right)$, and so $u$ vanishes identically on $\overline{\mathcal{V}\left(p_{0}\right)}$ (see, for instance, [8, Theorem 3.5]).

Thus, a standard reasoning allows to conclude that $u \equiv 0$ on $\Sigma$, namely $\nu \equiv 0$ on $\Sigma$. The proof finishes using Theorem 4 and Example 3.

Remark 7 The minimal cylinders described in Example 3 are not stable in general. For instance, for the simplest case of products $(f \equiv 1)$, when the fiber $M$ is compact such cylinders are stable if, and only if, $\gamma$ is a closed totally geodesic curve in $M[10]$.

Next we provide a geometrical condition to conclude that $f^{\prime \prime}(\tau)$ vanishes identically on the surface

Proposition 8 Let $\bar{M}=I \times_{f} M$ be a 3-dimensional Riemannian warped product such that $\left(M, g_{M}\right)$ is a complete Riemannian surface whose Gaussian curvature is bounded from below and the smooth function $f: \mathbb{R} \longrightarrow(0,+\infty)$ satisfies that $(\log f)^{\prime \prime} \geq 0$ and $\inf f>0$.

Then every complete minimal two-sided surface immersed in $\bar{M}$ contained in a slab, with $\nu \geq 0$, satisfies that $f^{\prime \prime}(\tau)=0$.

Proof: Let us take $\lambda=\inf f>0$. Using (8) and (9), we get

$$
\begin{aligned}
\triangle \log (\bar{g}(\xi, N)+\lambda)= & \frac{1}{(\bar{g}(\xi, N)+\lambda)^{2}}\left\{-\frac{1}{2}|A|^{2}\left(f(\tau)^{2}-\bar{g}(\xi, N)^{2}\right)-\bar{g}(\xi, N)(\bar{g}(\xi, N)+\lambda)|A|^{2}\right. \\
& \left.-\bar{g}(\xi, N)(\bar{g}(\xi, N)+\lambda)\left(1-\nu^{2}\right)\left(\frac{K^{M} \circ \pi_{M}}{f^{2}(\tau)}+(\log f)^{\prime \prime}(\tau)\right)\right\} .
\end{aligned}
$$

Since $f(\tau) \geq \lambda$, we have that

$$
\frac{1}{2}|A|^{2}\left(f(\tau)^{2}-\bar{g}(\xi, N)^{2}\right)+\bar{g}(\xi, N)(\bar{g}(\xi, N)+\lambda)|A|^{2} \geq \frac{1}{2}(\bar{g}(\xi, N)+\lambda)^{2}|A|^{2}
$$

and so, from (15), it follows that

$$
\triangle \log (\bar{g}(\xi, N)+\lambda) \leq \frac{\bar{g}(\xi, N)}{\bar{g}(\xi, N)+\lambda}\left(1-\nu^{2}\right)\left(\frac{K^{M} \circ \pi_{M}}{f^{2}(\tau)}+(\log f)^{\prime \prime}(\tau)\right)-\frac{1}{2}|A|^{2} .
$$

Now, consider on $\Sigma$ the metric, conformal to the induced metric $\langle$,$\rangle , given by$

$$
\widehat{g}=(\bar{g}(\xi, N)+\lambda)^{2}\langle,\rangle,
$$

which is complete because $\langle$,$\rangle is complete and \lambda>0$. Let us denote by $\widehat{K}$ the Gaussian curvature of $\Sigma$ furnished with the metric $\widehat{g}$.

From the well-known relation

$$
\left.(\bar{g}(\xi, N)+\lambda)^{2} \widehat{K}=K-\triangle(\bar{g}(\xi, N)+\lambda)\right)
$$


we obtain that

$$
\begin{aligned}
(\bar{g}(\xi, N)+\lambda)^{2} \widehat{K} \geq & -\frac{f^{\prime}(\tau)^{2}}{f(\tau)^{2}}-(\log f)^{\prime \prime}(\tau)\left(1-\nu^{2}\right)\left(1+\frac{\bar{g}(\xi, N)}{\bar{g}(\xi, N)+\lambda}\right) \\
& +\frac{K^{M} \circ \pi_{M}}{f^{2}(\tau)}\left(1-\left(1-\nu^{2}\right) \frac{\lambda}{\bar{g}(\xi, N)+\lambda}\right),
\end{aligned}
$$

where we have used (14), (8) and (16). In particular, since $K^{M}$ is bounded from below, then so is $\widehat{K}$.

We will use the following generalized maximum principle for Riemannian manifolds given by Omori [11] (see also Yau's paper [16]):

Let $\Sigma$ be a complete Riemannian manifold whose Ricci curvature is bounded away from $-\infty$ and let $u: \Sigma \longrightarrow \mathbb{R}$ be a smooth function on $\Sigma$.

a) If $u$ is bounded from above on $\Sigma$, then for each $\varepsilon>0$ there exists a point $p_{\varepsilon} \in \Sigma$ such that

$$
\left|\nabla u\left(p_{\varepsilon}\right)\right|<\varepsilon, \quad \Delta u\left(p_{\varepsilon}\right)<\varepsilon, \quad \sup u-\varepsilon<u\left(p_{\varepsilon}\right) \leq \sup u ;
$$

b) If $u$ is bounded from below on $\Sigma$, then for each $\varepsilon>0$ there exists a point $p_{\varepsilon} \in \Sigma$ such that

$$
\left|\nabla u\left(p_{\varepsilon}\right)\right|<\varepsilon, \quad \Delta u\left(p_{\varepsilon}\right)>-\varepsilon, \quad \inf u \leq u\left(p_{\varepsilon}\right)<\inf u+\varepsilon .
$$

Here $\nabla u$ and $\Delta u$ denote, respectively, the gradient and the Laplacian of $u$.

Let us denote by $\widehat{\Delta}$ the Laplacian operator on $(\Sigma, \widehat{g})$. Then we have that

$$
\widehat{\Delta} \tau=\frac{1}{(\lambda+\bar{g}(\xi, N))^{2}} \Delta \tau \text {. }
$$

As we are assuming that the surface $\Sigma$ is contained in a slab, the smooth function $\tau$ is, in particular, bounded from below. Hence, from the generalized maximum principle we know that for each $m \in \mathbb{N}$ there exists a point $p_{m} \in \Sigma$ such that

$$
-\frac{1}{m}<\widehat{\Delta} \tau\left(p_{m}\right) .
$$

Then, letting $m \rightarrow \infty$ and using (7) and (17), we obtain that $\frac{f^{\prime}(\inf \tau)}{f(\inf \tau)} \geq 0$. Reasoning analogously, since $\tau$ is bounded from above we obtain that $\frac{f^{\prime}(\sup \tau)}{f(\sup \tau)} \leq 0$. Therefore, by using also that $(\log f)^{\prime \prime} \geq$ 0 , it follows that $f^{\prime}(\tau)=0$ and the thesis follows immediately.

Since there are not complete cylinders contained between two slices, from Theorem 6 and Proposition 8 we conclude

Theorem 9 Let $\bar{M}=I \times_{f} M$ be a 3-dimensional Riemannian warped product such that $\left(M, g_{M}\right)$ is a complete Riemannian surface whose Gaussian curvature is bounded from below and the smooth function $f: \mathbb{R} \longrightarrow(0,+\infty)$ satisfies that $(\log f)^{\prime \prime} \geq 0$ and $\inf f>0$.

Then every complete minimal two-sided surface $\psi: \Sigma \longrightarrow \bar{M}$ contained in a slab, with $\nu \geq 0$, is stable. 
As a consequence of the following well-known result due to Schoen [13],

every complete stable minimal surface immersed a 3-dimensional Riemannian manifold with non-negative Ricci curvature is totally geodesic

we have the following Bernstein type result:

Theorem 10 Let $\Sigma$ be a complete minimal two-sided surface immersed in a 3-dimensional Riemannian warped product $\bar{M}=I \times_{f} M$ with non-negative Ricci curvature, such that $\nu \geq 0$ (resp. $\nu>0$ ). If $f^{\prime \prime}(\tau)=0$ (resp. $f^{\prime \prime}(\tau) \geq 0$ ) on $\Sigma$, then $\Sigma$ is totally geodesic.

Proof: It follows from the aforementioned Schoen's result, Theorem 6 and the fact that the minimal cylinders $C(J, \gamma)$ are totally geodesic.

Let $\bar{M}=I \times{ }_{f} M$ be a 3-dimensional Riemannian warped product. In the particular case when the positive function $f$ is linear, $f(t)=\alpha t+\beta$, with $\alpha \neq 0$, it must be $I \subseteq\{t \in \mathbb{R}: \alpha t+\beta>0\}$. Hence, for this kind of warping function it is $I \neq \mathbb{R}$ and there are no complete minimal cylinders as those described in Example 3. Therefore, under these assumptions we can conclude that every complete minimal two-sided surface such that $\nu \geq 0$ is stable.

Observe that, from (10), the Ricci curvature of $\bar{M}$ is given by

$$
\overline{\operatorname{Ric}}(X)=\frac{1-\bar{g}(X, T)^{2}}{f(t)^{2}}\left(K^{M}-f^{\prime}(t)^{2}\right)-\frac{f^{\prime \prime}(t)}{f(t)}(1+\bar{g}(X, T))
$$

for all unitary vector field $X \in \mathfrak{X}(\bar{M})$. If we assume that $f(t)=\alpha t+\beta$ ( $\alpha$ could be zero) and $K^{M} \geq \alpha^{2}$, then $\bar{M}$ has non-negative Ricci curvature. In this scenario, from Theorem 10, we get that every complete minimal two-sided surface with $\nu \geq 0$ is totally geodesic.

In the particular case when $\Sigma$ is compact, since $\bar{g}(\xi, N)$ is a non-negative function whose Laplacian is non-positive (see $(8)$ ), then $\bar{g}(\xi, N)$ is constant. We can reach the same conclusion if $\Sigma$ is complete and non-compact. In fact, using that the Gaussian curvature $K$ of $\Sigma$ is given by $($ see $(14))$

$$
K=\frac{\bar{g}(\xi, N)^{2}}{f(\tau)^{4}}\left(K^{M}-\alpha^{2}\right),
$$

which is non-negative because $K^{M} \geq \alpha^{2}$, from a classical result by Ahlfors and Blanc-FialaHuber [9] we conclude that $\Sigma$ is parabolic and reasoning as above we get that $\bar{g}(\xi, N)$ is constant.

So we have

Theorem 11 Let $\Sigma$ be a complete minimal two-sided surface immersed in a 3-dimensional Riemannian warped product $\bar{M}=I \times_{f} M$ such that $f(t)=\alpha t+\beta$ and $K^{M} \geq \alpha^{2}$. If $\nu \geq 0$ then $\Sigma$ is totally geodesic and $\bar{g}(\xi, N)$ is constant.

Remark 12 At this point, it worths pointing out some specific features of the cases $\alpha \neq 0$ and $\alpha=0$. 
- If $\alpha \neq 0$, then the totally geodesic surface $\Sigma$ cannot be a slice of $\bar{M}$. In fact, the slices $\Sigma_{t}=\{t\} \times M, t \in I$, have non-zero constant mean curvature $H_{t}=\frac{\alpha}{\alpha t+\beta}$. This fact will be used later in Theorem 13.

- If $\alpha=0$ and $\Sigma$ is compact, it is easy to reason that $\Sigma$ is a slice. In particular, as a consequence the fiber $M$ is also compact.

On the other hand, if $\alpha=0$ and $\Sigma$ is complete (non-compact), again from Theorem 6 we know than either $\nu \equiv 0$ and so $\Sigma$ is a cylinder, or $\nu \equiv$ cte $>0$. In the last case, if there exists a point $q \in M$ where $K^{M}(q)>0$, then $\Sigma$ is a slice. In fact, under this assumption, from $\triangle \bar{g}(\xi, N)=0$ it follows that the constant function $\nu$ must be 1 on $\Sigma$. A standard reasoning using [12, Lemma 14, chapter 4$]$ allows to conclude that $\Sigma$ is a slice. In particular the fiber $M$ is also complete. Note that the assumption $K^{M}(q)>0$ at some point cannot be removed, as shows the classical Bernstein's theorem in $\mathbb{R}^{3}$.

Given $\left(M, g_{M}\right)$ a Riemannian surface, for each $u \in C^{\infty}(M)$ let $\Sigma_{u}=\{(u(p), p): p \in M\}$ be the entire graph defined by $u$ on $M$. The subset $\Sigma_{u}$ can be seen as a regular surface in $\bar{M}=I \times{ }_{f} M$, where $I$ denotes an open interval with $u(M) \subset I$, and $f: I \longrightarrow \mathbb{R}$ is a positive smooth function. This graph inherits from $\bar{M}$ a Riemannian metric $g_{u}$ which, on $M$, is given by

$$
g_{u}=d u^{2}+f(u)^{2} g_{M} .
$$

The graph $\Sigma_{u}$ is minimal if and only if the function $u$ satisfies the following nonlinear elliptic equation in divergence form

$$
\operatorname{div}\left(\frac{D u}{f(u) \sqrt{f(u)^{2}+|D u|^{2}}}\right)=\frac{f^{\prime}(u)}{\sqrt{f(u)^{2}+|D u|^{2}}}\left\{2-\frac{|D u|^{2}}{f(u)^{2}}\right\},
$$

where $D u$ denotes the gradient of $u$ in $\left(M, g_{M}\right)$ and $|D u|^{2}=g_{M}(D u, D u)$.

As a consequence of Theorem 11 and Remark 12 we get the following non-existence result for Equation (19):

Theorem 13 Let $\left(M, g_{M}\right)$ be a Riemannian surface and $f(t)=\alpha t+\beta$ a non-constant linear function. If the Gaussian curvature of $M$ is such that $K^{M} \geq \alpha^{2}$, being the inequality strict at some point $q \in M$, then there is no entire solutions to the nonlinear elliptic equation (19).

Proof: Let us suppose that there exists such a solution $u$, and let $\Sigma_{u}$ be the graph in $\bar{M}$ associated to $u$. From Theorem 11 we know that $\bar{g}(\xi, N)$ is a positive constant.

Let $q \in M$ be a point where $K^{M}(q)>\alpha^{2}$ and take $\mathcal{O} \subset M$ an open subset such that $q \in \mathcal{O}$ and $K^{M}(p)>\alpha^{2}$ for all $p \in \mathcal{O}$. Now, since $\Delta \bar{g}(\xi, N)=0$ we obtain, using (8), that the graph on $\mathcal{O}$ must be contained in a slice with non-zero mean curvature, which is a contradiction.

\subsection{Special features for compact surfaces}

Let $\Sigma$ be a compact minimal two-sided surface immersed in a 3-dimensional Riemannian warped manifold $\bar{M}=I \times_{f} M$. As commented in Section 2, $\Sigma$ is stable if the quadratic form $Q$ given in (1) satisfies that $Q(\eta, \eta) \geq 0$ for all $\eta \in \mathcal{C}_{0}^{\infty}(\Sigma)=\mathcal{C}^{\infty}(\Sigma)$. 
From Lemma 2, if we take $\eta=\bar{g}(\xi, N)$ then

$$
Q(\bar{g}(\xi, N), \bar{g}(\xi, N))=\int_{\Sigma} 2 \frac{f^{\prime \prime}(\tau)}{f(\tau)} \bar{g}(\xi, N)^{2} .
$$

In particular, we get

Theorem 14 There is no any compact stable minimal two-sided surface in a 3-dimensional Riemannian warped manifold $\bar{M}=I \times_{f} M$ with $f^{\prime \prime}<0$.

Note that in the theorem above we do not need that the function angle $\nu$ does not change sign.

On the other hand, observe that if the warped function $f$ is such that $f^{\prime \prime} \leq 0$, then for a compact stable minimal surface in $\bar{M}=I \times_{f} M$ the integral (20) is zero and so $L \bar{g}(\xi, N)=0$. From Theorem 6 we know that it must be $\bar{g}(\xi, N)>0$, and consequently $f^{\prime \prime}(\tau)$ vanishes identically. Thus, we have proved the following result

Theorem 15 Let $\Sigma$ be a compact minimal two-sided surface with $\nu \geq 0$ immersed in a 3-dimensional Riemannian warped product $\bar{M}=I \times_{f} M$ with $f^{\prime \prime} \leq 0$. Then, $\Sigma$ is stable if, and only if, $f^{\prime \prime}(\tau)=0$ on $\Sigma$.

Remark 16 Assume that the fiber $M$ is compact. As is well-known, under this assumption the slices are compact. Assume also that $f^{\prime \prime} \leq 0$ and that

$$
\Omega=\left\{t \in I: f^{\prime \prime}(t)=0\right\}
$$

is a set of isolated points. Then, for every $t_{0} \in \Omega$ such that $f^{\prime}\left(t_{0}\right)=0$, the minimal slice $\Sigma_{t_{0}}=$ $\left\{t_{0}\right\} \times M$ is stable.

\subsection{Einstein Riemannian Warped Products}

Let $\bar{M}=I \times_{f} M$ be a Riemannian warped product of a $n$-dimensional Riemannian manifold $\left(M, g_{M}\right)$ and $\left(I, d t^{2}\right)$, with $f$ a positive real function on $I$. It is not difficult to check (see [3, Corollary 9.107]) that $(\bar{M}, \bar{g})$ is Einstein with $\overline{\operatorname{Ric}}=\bar{c} \bar{g}$ if, and only if, $\left(M, g_{M}\right)$ has constant Ricci curvature $c$ and $f$ satisfies the differential equation

$$
-\frac{f^{\prime \prime}}{f}=\frac{\bar{c}}{n} \quad \text { and } \quad-\frac{\bar{c}(n+1)}{n}=\frac{(n-1) f^{\prime 2}-c}{f^{2}} .
$$

It is a straightforward computation to integrate the solutions of (21) to obtain the following:

Lemma 17 The only solutions to Equation (21) are

- $\bar{c}=0, c=0$ and $f(t)=a>0$ cte.

- $\bar{c}=0, c>0$ and $f(t)=\varepsilon \sqrt{\frac{c}{n-1}} t+b$, where $\varepsilon= \pm 1$ and $b \in \mathbb{R}$. 
In particular, since $f^{\prime \prime}=0$ for all the solutions to Equation (21), we are under the hypotheses of Theorem 6 and we could rewrite this result for surfaces immersed in a 3-dimensional Einstein Riemannian warped product.

Observe that in a 3-dimensional Einstein Riemannian warped product $\bar{M}=I \times_{f} M$ with $f(t)=\alpha t+\beta$, we have that $K^{M} \equiv \alpha^{2}$. In particular, from (18), every totally geodesic surface in such an ambient space is flat. So we have:

Corollary 18 Every complete minimal two-sided surface with $\nu \geq 0$ immersed in a 3-dimensional Einstein Riemannian warped product $\bar{M}=I \times_{f} M$ is flat.

\section{Acknowledgments}

The first author is partially supported by the Spanish MICINN Grant with FEDER funds MTM201343970-P and by the Junta de Comunidades de Castilla-La Mancha Grant PEII-2014-001-A. The second author is partially supported by the Spanish MICINN Grant with FEDER funds MTM201347828-C2-1-P and by the Junta de Andalucía Regional Grant with FEDER funds P09-FQM-4496.

\section{References}

[1] L.J. Alías, J.H.S. de Lira and J.M. Malacarne, Constant higher-order mean curvature hypersurfaces in Riemannian spaces, J. Inst. Math. Jussieu 5 (2006), no. 4, 527-562.

[2] J.L. Barbosa, and M. do Carmo, On the size of a stable minimal surface in $\mathbb{R}^{3}$, Amer. J. Math. 98 (1976), no. 2, 515-528.

[3] A.L. Besse, Einstein Manifolds, Springer-Verlag, Berlin 1987.

[4] R.L. Bishop and B. O'Neill, Manifolds of negative curvature, Trans. Amer. Math. Soc. 145 1969 1-49.

[5] M. do Carmo and C.K. Peng, Stable complete minimal surfaces in $\mathbb{R}^{3}$ are planes, Bull. Amer. Math. Soc. 1 (1979), no. 6, 903-906.

[6] T.H. Colding and W.P. Minicozzi II, A Course in Minimal surfaces, Graduate Studies in Mathematics 121, AMS, Providence 2011.

[7] D. Fisher-Colbrie and R. Schoen, The structure of complete stable minimal surfaces in 3manifolds of nonnegative scalar curvature, Comm. Pure Appl. Math. 33 (1980), 199-211.

[8] D. Gilbarg and N.S. Trudinger, Elliptic partial differential equations of second order, Vol. 224, Springer, 2001.

[9] J.L. Kazdan, Parabolicity and the Liouville property on complete Riemannian manifolds, Aspects of Math., E10, Ed. A.J. Tromba, Friedr. Vieweg and Sohn, Bonn (1987), 153-166.

[10] W.H. Meeks and H. Rosenberg, Stable minimal surfaces in $M \times \mathbb{R}$, J. Differential Geom. 68 (2004), no. 3, 515-534. 
[11] H. Omori, Isometric immersions of Riemannian manifolds, J. Math. Soc. Japan 19 (1967), 205-214.

[12] B. O’Neill, Semi-Riemannian Geometry with applications to Relativity, Academic Press, 1983.

[13] R. Schoen, Estimates for stable minimal surfaces in three dimensional manifolds, Annals Math. Studies 103, Princeton Univ. Press, Prienceton, 1983.

[14] R. Schoen and S.T. Yau, Existence of incompressible minimal surfaces and the topology of three-dimensional manifolds with nonnegative scalar curvature, Ann. of Math. 110 (1979), no. $1,127-142$.

[15] R. Schoen and S.T. Yau, On the structure of manifolds with positive scalar curvature, Manuscripta Math. 28 (1979), no. 1-3, 159-183.

[16] S.T. Yau, Harmonic functions on complete Riemannian manifolds, Commun. Pure Appl. Math. 28 (1975), 201-228. 